\title{
Simulation expérimentale de l'écoulement dans un talus de sol soumis à la marée
}

\section{A. REZZOUG}

A. ALEXIS

P. THOMAS

Laboratoire de Génie Civil, École Centrale, Nantes

1. rue de la Noë

44072 Nantes Cedex

Institut Universitaire

de Technologie,

58, rue Michel-Ange

BP 420

44606 Saint-Nazaire Cedex
Les fluctuations de la marée, comme toutes les contraintes océaniques, sollicitent la stabilité des ouvrages portuaires et les talus intertidaux en général. Cet effet dépend, entre autres, des vitesses de

l'écoulement et des pressions induites par la propagation de la marée dans le milieu de la masse de l'ouvrage. Le but de ce travail est tout d'abord d'étudier l'évolution du champ instantané de pression dans le massif de sol sous des conditions cycliques de variation de niveau d'eau par une simulation originale de la marée, puis d'élaborer une méthode rationnelle simple pour localiser à tout moment le niveau d'eau de la surface libre et quantifier la distribution du champ de pression dans le but d'une éventuelle étude de stabilité.

Cet article est consacré à une description d'un modèle physique de simulation de la marée et des exemples de résultats expérimentaux. Des comparaisons sont effectuées avec les résultats d'une modélisation numérique de la tranche de sol du modèle physique.

\section{Experimental simulation of flow in sloping soil under tide}

The stability of harbour structures and intertidal slopes in general, subjected to the effects of cyclic changing level by tide, is dependant on among other factors, the pore pressures and flow velocities induced within the medium of structure mass due to seepage.

This paper contains some examples of experimental results for tidal transient seepage obtained from the physical flow model and comparisons of this result with numerical results in case of physical model. It contains also, before all this, a description and exploitation of the model. The physical model made up a slice of soil submissive under cyclical variations of water table for an original tidal simulation.

The purpose of this study is to investigate the transient development of pore pressures in an earth bank under conditions of cyclical variation of sea level with tide and to evolve a more rational method for predicting location of free surface and distribution of pore pressures for use in design and stability analysis.
} 


\section{Introduction}

Dans les zones littorales soumises à de fortes marées, il apparait fréquemment des perturbations ou ruines d'ouvrages sous l'action des contraintes océaniques périodiques.

Dans le but d'estimer l'impact des actions hydrauliques cycliques dues à la marée sur le comportement des talus estuariens et sur les ouvrages portuaires en général, tels que les quais et les digues, nous avons cherché à mieux cerner le phénomène de l'écoulement à caractère dynamique dans ces types d'ouvrages.

Nous nous intéressons, ici, aux études du mouvement et de l'évolution de la surface libre. Pour la recherche de solutions nous avons fait appel à des modèles mathématiques et physiques permettant d'estimer le comportement hydrodymamique de l'écoulement à surface libre que nous souhaitons analyser et prédire.

Dans cet article, nous proposons un modèle phy sique du sol qui est une reconstitution d'un massif poreux de sable, en laboratoire à petite échelle, avec une simulation originale du mouvement de la marée.

Ce modèle physique permet d'obtenir des informations indispensables pour l'exploitation et la validation d'un modèle mathématique que nous décrirons ensuite brièvement.

Il convient de préciser ici, que notre objectif n'est pas la recherche d'une similitude physique de la nature, donc comme première approche, n'est pas la reproduction fidèle des conditions aux limites naturelles. Il s'agit simplement d'étudier expérimentalement le comportement d'une tranche de sol présentant une frontière oblique ou verticale avec une réserve d'eau dont le niveau varie d'une façon périodique. Il s'agit également de tester la validation du modèle mathématique que nous proposons par une application dans le cas spécifique correspondant à ce modèle physique; et déduire des informations utiles, éventuellement, une validation pour le cas réel en nature.

\section{2}

\section{Description et schématisation du phénomène naturel}

\section{Description du phénomène naturel}

Sous laction des contraintes cycliques induites par la marée, les talus portuaires se remplissent et se vident périodiquement.

L'onde marée se propage à l'intérieur de ces talus et donne naissance à des phénomènes d'écoulement dans les sols alternativement immergés et émergés.

Cet écoulement se caractérise par sa surface libre, (ensemble des points à la pression atmosphérique), audessus de laquelle se situe la frange capillaire.

Le talus a une pente oblique ou verticale. Constitué d'un sol perméable, il repose sur une couche généralement imperméable.
A l'intérieur du talus, assez loin de l'interface mertalus, l'impact de l'onde de marée diminue, l'amplitude des oscillations se réduit, et il apparaît un niveau d'équilibre.

Les vitesses d'écoulement pourront causer des effets perturbateurs sur la stabilité globale (possibilité de grand glissement) ou locale (affouillement) des massifs portuaires, soumis à la marée.

\section{2}

\section{Schéma de modélisation}

Ce phénomène naturel peut être schématisé ainsi, en désignant par PM et BM la pleine mer et la basse mer:

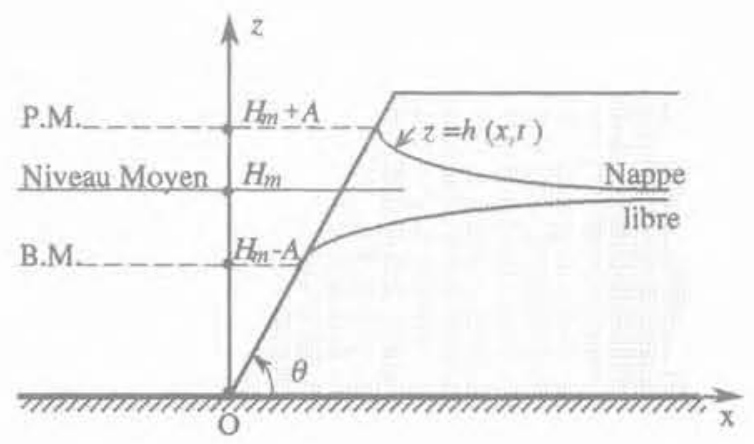

FG.1 Schéma du talus soumis au marnage. Scheme of the interdidal slope.

L'onde marée a un niveau moyen $\mathrm{H}_{\mathrm{m}}$, une amplitude A et une période $T$ correspondant à une pulsation $\omega$, supposés constants. Son niveau oscille verticalement selon la loi:

$$
h(0, t)=H_{m}+A \sin \omega t
$$

$h(x, t)$ désigne la cote de la surface libre à partir du substratum, supposé imperméable, sur lequel repose le talus de pente $P$.

\section{Modèle physique}

\section{1}

\section{Description}

Pour s'affranchir de la plupart des hypothèses habituelles, nous proposons un modèle physique expérimental bidimensionnel, original, base de cette étude, qui permet:

- d'effectuer un suivi de l'évolution de la surface libre; - de mesurer la perméabilité du massif en place. 




système d'entraînement

bac de mesures

FG.2. Schéma général du modèle physique expérimental.

$\rightarrow$ sens de l'écoulement 1 simulation du côté mer

+ prises de pression 2 simulation du côté terre

General scheme of experimental physical model.

\section{1.}

La figure 2 montre d'une façon globale les deux parties principales du montage de cette installation: le système excitateur (simulation de la marée), et le système récepteur (domaine de l'expérimentation).

Un mécanisme excitateur composé d'un moteur entrainant un entonnoir constamment en trop-plein, constitue le système d'entraînement (Fig. 3). L'entonnoir tourne en décrivant un cercle dans un plan vertical, et permet d'obtenir une variation sinusoidale du niveau d'eau dans le réservoir, côté mer, du bac de mesures recréant ainsi le phénomène de la marée.

Le domaine d'expérimentation est constitué, dans son ensemble, d'un bac de mesures de forme parallélépipédique de dimensions $1,3 \mathrm{~m} \times 1,18 \mathrm{~m} \times 0,2 \mathrm{~m}$ (Fig. 2). Ce bac repose sur une base horizontale imperméable et contient une tranche de sable fin (de granulométrie étalée entre 0 et $1 \mathrm{~mm}$ ) comprise entre deux parois latérales de plexiglas (rigidifiées par des profilés métalliques). Chaque paroi est également en contact avec un réservoir ayant la profondeur du bac, une section de $20 \mathrm{~cm} \times 20 \mathrm{~cm}$, et communique directement avec la tranche à travers des grilles. Nous avons appelé « réservoir côté mer », le réservoir amont où la simulation du phénomène de la marée est appliquée. Cette simulation est assurée par la communication directe de ce réservoir au système d'entraînement par l'intermédiaire d'un tube mou en plastique de $12 \mathrm{~cm}$ de diamètre. De même, nous avons appelé «réservoir côté terre» le réservoir du côté aval de la tranche où le niveau d'eau est laissé libre. Ce dernier est exploité pour l'observation et la simulation de la limite infinie.

Le bac de mesures est porté par un portique en profilés métalliques rigides, l'ensemble repose sur des cales de réglage de l'horizontalité du portique et de la verticalité des parois du bac.

Les mesures de charges sont effectuées à l'aide de tubes piézométriques connectés aux prises de pressions. Ces prises sont installées de manière à former un maillage quadrilatère sur toute une face du modèle.

Nous pouvons faire varier l'angle de l'inclinaison $\theta$ de l'interface eau-sol du côté mer de la tranche, dans le but de simuler la pente d'un talus.

Nous avons ainsi pu simuler les conditions aux limites naturelles en établissant une nappe à surface libre et en faisant varier le niveau pour représenter l'impact de la marée (A. Alexis et al., 1990).

Nous avons ainsi pu suivre l'évolution de la surface libre et le champ de pression dans la zone saturée lors des oscillations périodiques de la nappe.

\section{$3.1,2$}

La figure 4 décrit le montage de mesure de perméabilité $k$ de la tranche de sol en place. Nous avons 


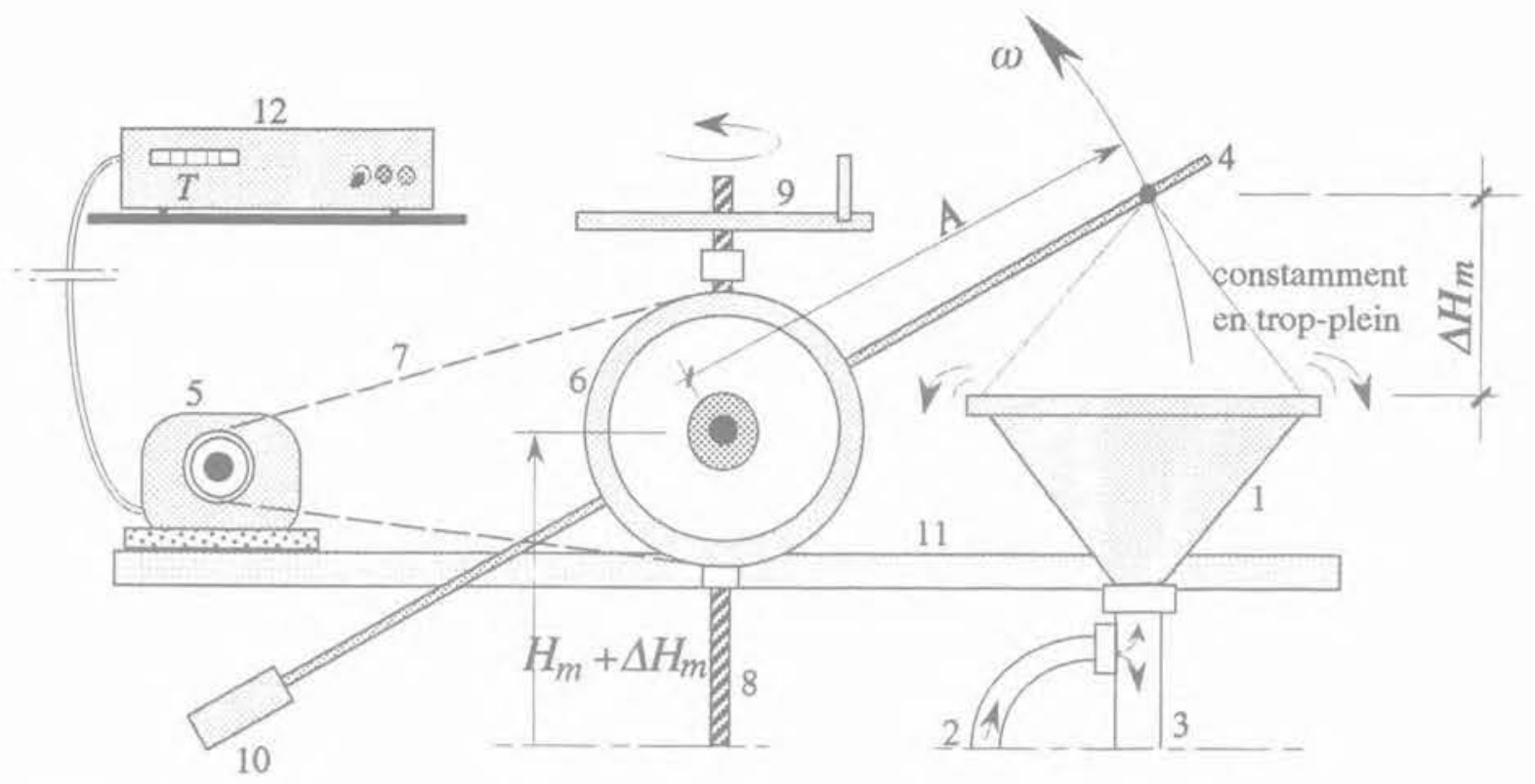

กิG. 3 Détails du mécanisme de simulation de la marée sinusoïdale par le système d'entrainement.

1) Entonnoir constamment en trop-plein, reçoit l'eau par 2, et la transmet au bac de mesures « côté mer » par 3. 2) Tube d'admission à la pompe. 3) Tube de transfert. 4) Tige support soudée à la poulie.

L'amplitude A de la marée peut être réglée en fonction de la position du point d'attache de l'entonnoir sur cette tige. 5) Moteur électrique pas à pas, entraine la poulie 6 par la courroie 7 . Il est commandé par le générateur 12. 6) Poulie. 7) Courroie. 8) Tige filetée, sa rotation règle la hauteur de la barre 11 et du centre de poulie 6: et donc la hauteur moyenne de la marée. 9) Manivelle de rotation manuelle de la vis 8. 10) Contrepoids, compense le poids de l'entonnoir. 11) Barre support pour le moteur 5 et la poulie 6. 12) Générateur d'impulsion. Il règle la période et pilote le moteur 5 . Mechanism details of the sinusoidal tidal simulation by dragging system.



FG.4 Schéma général d'un montage de mesure de perméabilité de la tranche de sol en place. General scheme of permeability measurement of a slice of soil in place. 
imposé une différence de charge entre les deux réservoirs en gardant la ligne de la surface libre peu inclinée sur l'horizontale. Soit $\mathrm{H}_{2}$ et $\mathrm{H}_{2}$ les hauteurs côté amont et aval de la tranche $\left(\mathrm{H}_{1}>\mathrm{H}_{2}\right)^{2}$. Nous mesurons le débit aval $\mathrm{Q}$ et nous en déduisons $\mathrm{k}$ par application de la loi de Dupuit pour les écoulements plans (Mahé, 1989):

$$
\mathrm{Q}=\frac{1}{2 L} \mathrm{k}\left(H_{1}^{2}-H_{2}^{2}\right)
$$

Pour ce sable nous avons obtenu une perméabilité $\mathrm{k}=8,77.10^{-4} \mathrm{~m} / \mathrm{s}$ (Fig, 5), et une porosité efficace moyenne de l'ordre de 0,12 , mesurée par la quantité d'eau libre dans trois échantillons préparés dans les mêmes conditions que la tranche.

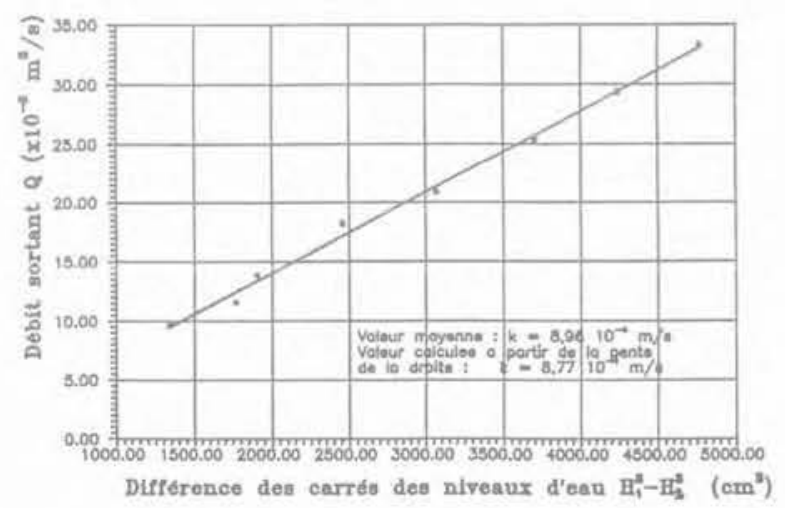

FG. 5 Courbe expérimentale de détermination de la perméabilité $k$.

Experimental curve of determination of permeability $\mathrm{k}$.

\section{2}

\section{Exploitation}

La présence de bulles d'air dans le circuit de l'écoulement constitue un problème délicat, systématiquement rencontré chez les expérimentateurs en hydraulique des sols. Dans notre cas, il s'est posé aussi bien dans le milieu de la tranche de sable qu'au niveau des prises de pression.

Ces bulles d'air, dont la taille est environ le diamètre des tubes piézométriques utilisês, perturbent les mesures et affectent la précision.

Pour faire face à ce probème, nous avons cherché à éliminer leur présence dans la tranche de sable. La méthode de pluviation uniforme du sable (recommandée pour la fabrication de massifs homogènes), dans le bac rempli d'eau lors de la mise en place de la tranche nous est parue bien adaptée: les grains se dispersent et chutent dans l'eau régulièrement alors que les bulles d'air remontent et disparaissent à la surface libre.

La réalisation des mesures nous a conduit à trois observations concernant:

- la périodicité : l'oscillation de l'onde incidente sinusoidale (côté mer), est transmise à la surface libre à l'intérieur du massif, qui oscille avec la même périodicité; - le niveau d'eau: il apparaît une surélévation dans le réservoir côté terre par rapport au niveau moyen à l'amont où le mouvement sinusoïdal du niveau d'eau est imposé ;

- la variation du niveau d'eau dans le réservoir côté terre: environ $0,5 \mathrm{~cm}$ d'amplitude par rapport à $16 \mathrm{~cm}$ dans le réservoir côté mer. Le sable utilisé est choisi pour la fabrication d'un modèle qui est considéré incompressible. La variation observée ne pose pas de problème pour la modélisation mathématique si nous ciblons une confrontation des résultats de ce modèle physique aux modèles mathématiques.

Pour chaque jeu de données expérimentales (période, amplitude, niveau moyen..., voir tableau I), les valeurs de pression recueillies sont représentées sous forme d'une carte de lignes équipotentielles, grâce à un programme d'interpolation. La position de la surface libre est calculée par la suite et mise en évidence sur la même carte.

Nous avons choisi, ici, de représenter quelques exemples de nos résultats expérimentaux. Les figures 6 et 7 montrent les cartes de lignes équipotentielles de lécoulement dans la tranche de sol de notre modèle physique. L'axe vertical donne la valeur de la charge hydraulique dans la zone saturée entre la base imperméable du modèle et le niveau de la surface libre, alors que l'axe horizontal donne la pénétration en $\mathrm{cm}$ dans la tranche. L'origine du repère est fixée à son coin inférieur du côté du réservoir côté mer. Les deux séries de cartes (Fig. 6 et 7) correspondent aux données figurant sur le tableau I à chaque quart de cycle.

TABIEAUI Récapitulation et comparaison des grandeurs $\mathrm{H}_{m^{\prime}} \mathrm{Ne}$, et Ne théorique dans trois cas.

Recapitulation and comparison of the parameters $\mathrm{H}_{\mathrm{m}}, \mathrm{Ne}$ and theoretical Ne for three cases.

\begin{tabular}{|c|c|c|c|c|c|c|}
\hline \multirow{3}{*}{$\begin{array}{l}T \\
\text { (s) }\end{array}$} & \multicolumn{6}{|c|}{ Niveau d'eau dans le réservoir (cm) } \\
\hline & \multicolumn{4}{|c|}{ Cóté mer } & \multicolumn{2}{|c|}{ Cóté terre } \\
\hline & $\mathrm{H}_{\max }$ & $\mathrm{H}_{\min }$ & $\mathrm{H}_{a}$ & A & $\mathrm{Ne}$ & Ne théor \\
\hline \multicolumn{7}{|c|}{ Ecran Vertical : Fig, 6 et 10} \\
\hline 80 & 98,7 & 66,3 & 82,5 & 16,2 & 83,3 & 83,3 \\
\hline \multicolumn{7}{|c|}{ Écran Vertical : Fig. 9} \\
\hline 120 & 98,3 & 66,7 & 82,5 & 15,8 & 83,3 & 83,3 \\
\hline \multicolumn{7}{|c|}{ Pente $=1:$ Fig. 7 et 11} \\
\hline 120 & 70,3 & 44,9 & 57,6 & 12.7 & 58,7 & 59,9 \\
\hline
\end{tabular}

Nous remarquons, à mi-flot, pendant la marée montante, une zone de perturbation, dépression dans le cas présent, due à la confrontation entre l'écoulement entrant et sortant au cours du cycle. Cette zone attire notre attention, et mérite de faire l'objet d'une analyse critique. Elle disparait en s'éloignant dans la tranche pendant le flot. Elle reprend naissance juste à côté du réservoir côté mer pendant le jusant, mais cette fois-ci en surpression, et disparaît également en s'éloignant de ce dernier vers le réservoir côté terre. C'est ainsi que cette zone de perturbation se crée près de l'interface massif-mer. Elle est donc sollicitée par des battements importants de fluctuations de pression.

Le fort rabattement de la ligne de surface libre ainsi que les rétrécissements des équipotentielles observés dans cette zone mettent en évidence l'importance des vitesses de filtration en particulier sur l'interface talus-mer.

Par contre en s'éloignant vers le côté terre de la tranche, nous constatons que l'écoulement devient plus laminaire et vérifie l'hypothèse légitime des nappes de Dupuit. Cela s'explique par la quasi-verticalité des 




(a) mi-flot mid flood

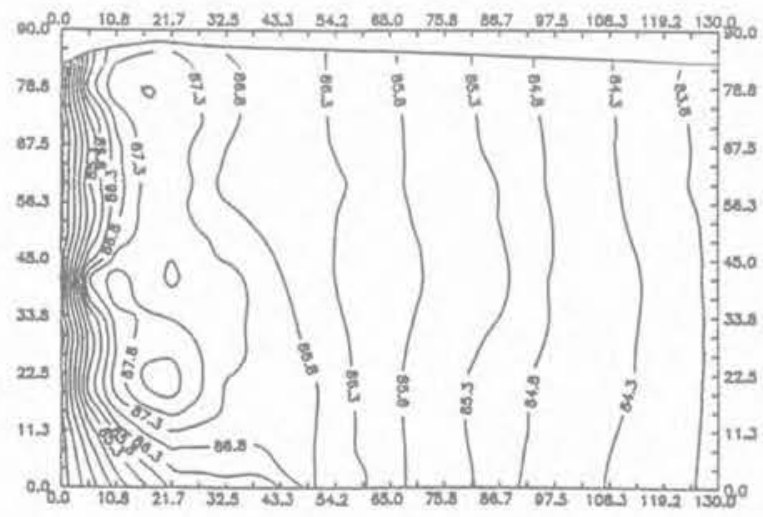

(c) mi-jusant mid ebb

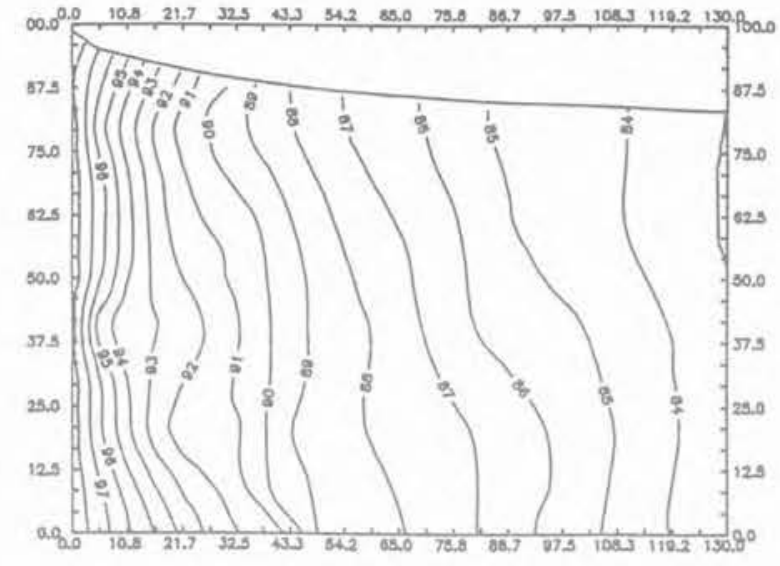

(b) pleine mer high tide

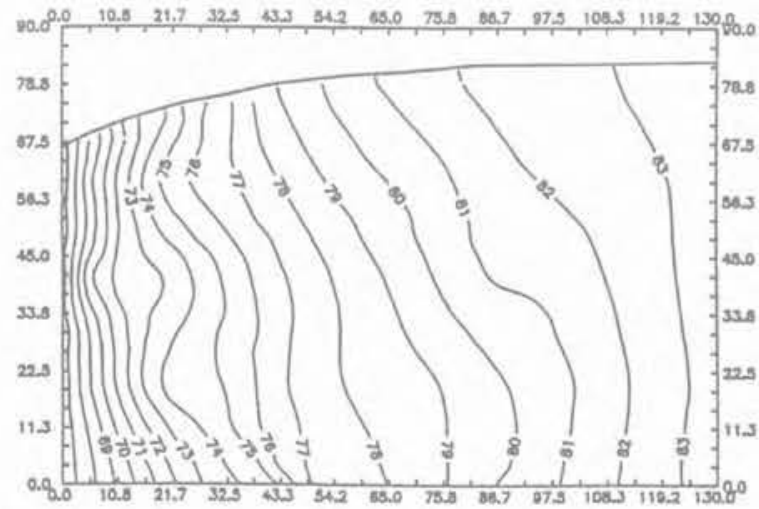

(d) basse mer low tide

FIG.6 Lignes équipotentielles et surface libre (écran vertical),

Equipotential lines and free surface (vertical bank).

lignes équipotentielles. Ces dernières s'interrompent à leur niveau pour créer ainsi la ligne de la surface libre.

Dans les deux séries de cartes, et malgré un temps de cycle réduit, un niveau d'eau stationnaire dans le réservoir côté terre, reste difficile à réaliser, vu la forte perméabilité de la tranche. Nous soulignons ici que ce niveau oscille au-dessus de la hauteur moyenne de la marée simulée. Des détails d'une justification analytique de la surélévation du niveau d'eau dans les talus estuariens, sont déjà donnés (Rezzoug et al., 1993', $\left.1993^{2}\right)$. De même des constatations en nature concernant ce phénomène ont été observées et semblent incontestables pour De Cazenove (1971); néanmoins, sa justification nous semble loin d'être effective dans la mesure où il attribue cette constatation à d'autres facteurs parasites (présence de la vase sur la pente, dissymétrie des marées...). Une telle justification expérimentale sur un modèle physique à petite échelle est un des objectifs de cette étude. Cette remontée du niveau d'eau dans les ouvrages portuaires des ports à marée, mérite d'être prise en compte dans les calculs de conception et de vérification de stabilité.

\section{Confrontation théorie-expérimentation}

\section{1}

\section{Nos études analytiques antérieures}

Ce modèle physique a été exploité pour permettre une analyse de validité d'un modèle mathématique des écoulements transitoires de nappes libres dans un massif intertidal, simulant le comportement des talus portuaires sollicités par la marée.

Cette modélisation mathématique est basée sur la résolution de l'équation de Dupuit en transitoire non linéaire donnée par Polubarinova (1962):

$$
\frac{\partial h(x, t)}{\partial t}=\frac{k}{2 n} \frac{\partial^{2} h^{2}(x, t)}{\partial x^{2}}
$$




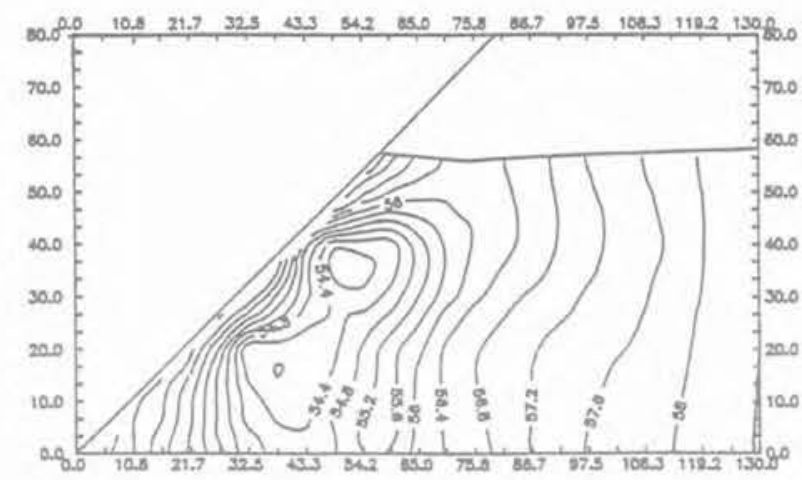

(a) mi-flot mid flood

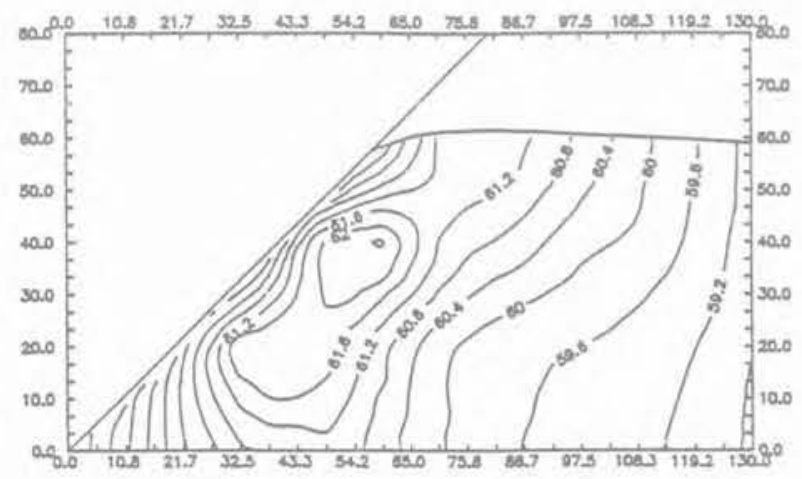

(c) mi-jusant mid ebb

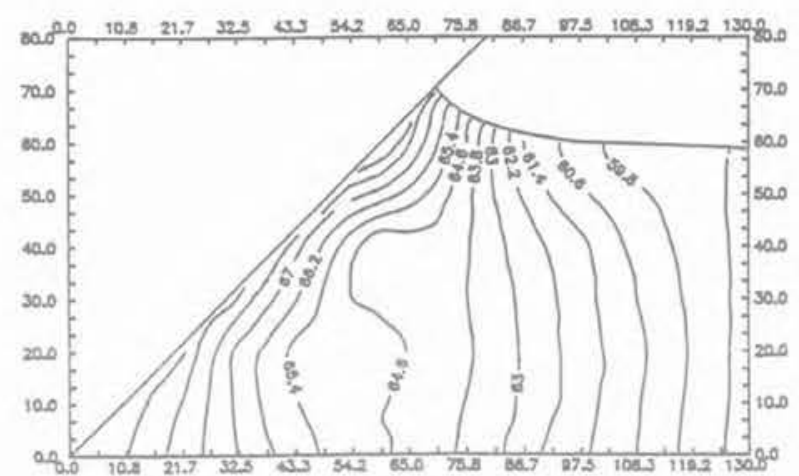

(b) pleine mer high tide



(d) basse mer low tide

FG.7 Lignes équipotentielles et surface libre (talus de pente unité).

Equipotential lines and free surface (unit slope).

Elle a été décrite en s'appuyant sur la loi de Darcy et l'hypothèse de Dupuit dans un milieu homogène poreux, saturé, perméable et indéformable. Ces hypothèses sont tout à fait applicables au modèle physique proposé ici. Nous avons donné par ailleurs (Rezzoug et al., 1993') la fonction analytique $h(x, t)$ qui décrit l'allure de la surface libre dans le cas général d'un talus naturel semi-émergé de perméabilité k et de porosité n. Un niveau d'eau dans le réservoir côté terre stable, se traduit par une vitesse d'infiltration nulle entre la tranche et le réservoir. Cette condition représente alors, par analogie, la condition naturelle à l'infini.

Les études analytiques et numériques (Alexis, 1987; Rezzoug, 1994) ont montré une onde progressive d'écoulement pénétrant à l'intérieur du talus. Elle s'amortit sur une distance fonction de la perméabilité du milieu. Cette onde caractérise la longueur d'une zone perturbée aussi bien par les changements instantanés et cycliques de la pression interstitielle que par les vitesses d'écoulement induites par la marée. Les calculs mettent également en évidence une surcote du niveau d'équilibre ( $\mathrm{Ne}$ ) dans le talus par rapport au niveau moyen de la marée $\left(\mathrm{H}_{\mathrm{m}}\right)$.
La variation du niveau d'eau dans le réservoir côté terre et son influence dans l'alimentation et le drainage de la nappe (dans la tranche), ne peut être négligée. Donc, pour réaliser une confrontation exacte et objective entre les résultats du modèle mathématique et T'expérimentation, il faut prendre en compte, dans les calculs, la fluctuation du niveau d'eau dans ce réservoir.

\section{2}

\section{Simulation numérique de l'essai en laboratoire}

Pour pouvoir comparer les résultats expérimentaux et théoriques, nous avons utilisé le moyen des différences finies pour modéliser la tranche de sol ainsi que ses conditions aux limites physiques. Pour simuler la marée, le niveau d'eau oscille dans le premier réservoir selon la loi sinusoïdale (1).

La discrétisation en différences finies selon un schéma explicite centré de l'équation non linéaire (2), avec $\mathrm{x}=\mathrm{i} \Delta \mathrm{x}$ et $\mathrm{t}=\mathrm{j} \Delta \mathrm{t}$, (Alexis, 1987) donne: 


$$
h_{i, j+1}=h_{i, j}+K\left[h_{i+1, j}^{2}-2 h_{i, j}^{2}+h_{i-1, j}^{2}\right]
$$

avec:

$$
K=\frac{k}{2 n} \frac{\Delta t}{\Delta x^{2}}
$$

L'étude de stabilité des calculs donne une condition nécessaire $\left(K \leq \frac{1}{4 A}\right)$ (Rezzoug, 1990).

Dans l'autre réservoir, le niveau d'eau est laissé libre. Pendant un intervalle de temps $\Delta t$ la quantité d'eau communiquée entre la tranche et le réservoir de largeur $D$, est égale à la variation $\Delta \mathrm{h}$ du niveau d'eau dans ce dernier (Fig. 8). La vitesse d'infiltration selon la loi de Darcy $\vec{v}=-k \overrightarrow{g r a d h}$ au temps t est:

$$
v h_{n, j}=\frac{\Delta h}{\Delta t} D
$$

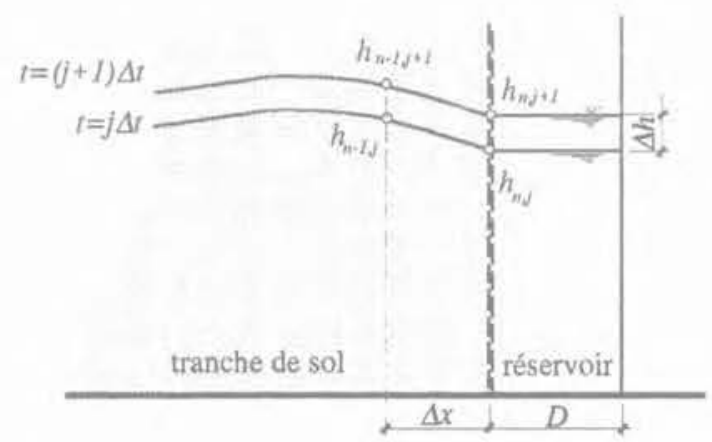

FIG.8 Modélisation de l'évolution du niveau d'eau, dans le second réservoir en fonction de la variation de la surface libre de la nappe dans la tranche de sol Modelisation of water level evolution in the second reservoir versus the free surface change of table water in the slice of soil.

Le découpage de la tranche en $n$ points répartis le long de sa largeur permet une discrétisation de la grandeur $h$ surr l'axe des $x$. Au point $n$ et à l'instant $t=j D t$, elle est définie par $h_{\mathrm{n}, \gamma^{-}}$La variation $\Delta \mathrm{h}$ est alors:

$$
\Delta h=\frac{k}{D} \frac{h_{n-1, j}-h_{n, j}}{\Delta x} h_{n, j} \Delta t
$$

On en déduit au temps $(j+1) \Delta t$ :

$$
h_{n, j+1}=h_{n, j}+\frac{k}{D} \frac{h_{n-t, j}-h_{n, j}}{\Delta x} h_{n, j} \Delta t
$$

Nous avons réalisé un programme, appelé Mareesol, de calcul des écoulements transitoires dans un massif saturé semi-émergé, soumis à la marée. Au sein de ce programme, le module MSDIF réalise lá résolution de l'équation (8) correspondant à cette discrétisation en différences finies.

\section{3}

\section{Comparaison des résultats théoriques et expérimentaux}

Les figures 9, 10 et 11 visualisent une comparaison entre les profils de surfaces libres expérimentales et



FIG. 9 Comparaisons des profils de surface libre. Écran vertical.

Comparisons of profiles of free surface. Vertical bank.

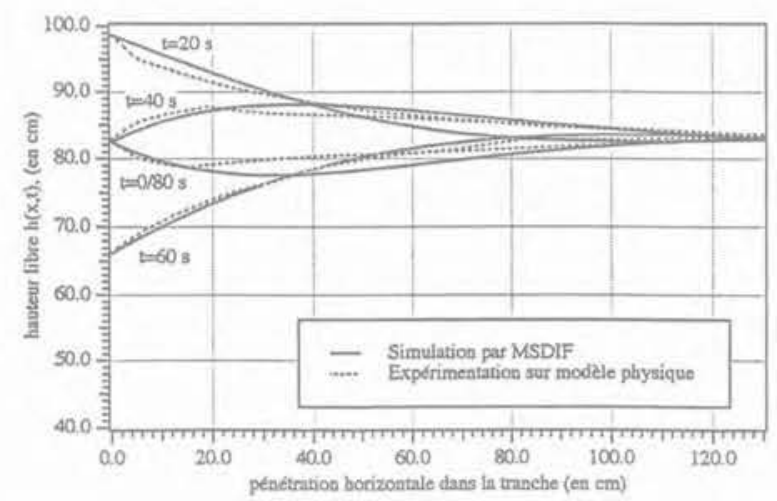

FIG 10 Comparaisons des profils de surface libre. Écran vertical.

Comparisons of profiles of free surface. Vertical bank.

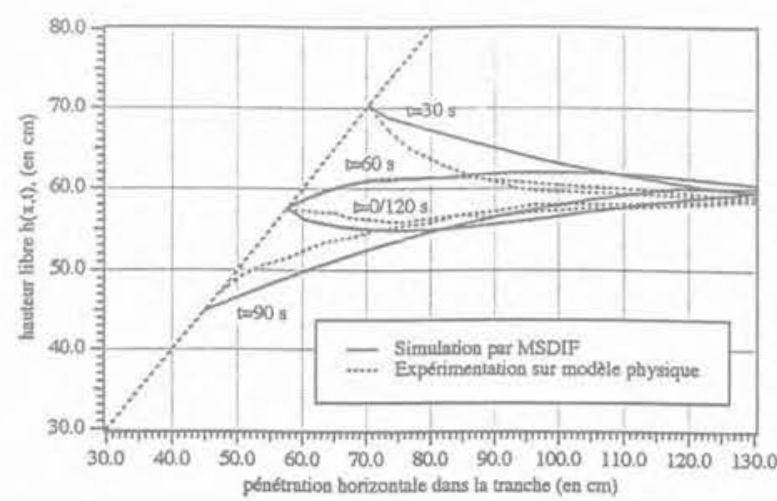

FIG. 11 Comparaisons des profils de surface libre. Pente unité.

Comparisons of profiles of free surface. Unit slope. 
théoriques. Elles représentent respectivement les cas d'un massif vertical et le cas d'un talus de pente. Nous avons présenté un couple de profils (théorique-expérimental), à chaque quart du cycle. Le modèle expérimental, ici, est toujours la tranche de sable décrite précédemment, avec une simulation d'une marée par une variation sinusoïdale accélérée du niveau d'eau dans le réservoir côté mer, alors que le modèle mathématique est décrit par l'équation (4), compte tenu des conditions aux limites (8) et (1) appliquées à la tranche expérimentale.

La surélévation du niveau d'êquilibre est exprimée de la même façon chez les deux modèles sur les figures 9 et 10 et plutôt légèrement surestimée par le modèle mathématique sur la figure 11. Nous considérons que ces deux figures quantifient la tolérance des résultats du modèle mathématique proposé par les résultats de notre modèle physique. Les résultats synthétisés dans ses deux figures visualisent la validation du modèle mathématique proposé au moyen du modèle physique que nous avons réalisé (Rezzoug et al., $1993^{2}$ ).

11 serait également intéressant de confronter ces résultats expérimentaux à d'autres théories existantes dans la littérature, comme la théorie des milieux non saturés. Ces travaux devront donner plus de détail, si nous envisageons aussi des manipulations sur des sols de granulométrie différentes.

\section{5}

\section{Synthèse et conclusion}

L'écoulement périodique dans un massif soumis à la marée constitue un phénomène naturel complexe en raison de la difficulté de l'estimation de l'interaction Hydraulique-Géotechnique dans un milieu poreux soumis à des sollicitations cycliques.

Une meilleure connaissance de ces problèmes hydrauliques des écoulements internes permet de mieux appréhender l'étude des problèmes de génie civil, tels que la conception et le dimensionnement des ouvrages:
Le modèle physique proposé permet d'obtenir une série d'informations dont l'exploitation fournit d'utiles indications pour l'utilisation et la validation des modèles mathématiques qui reposent, généralement, sur des hypothèses dont le domaine de validité n'est pas toujours parfaitement adapté.

Le niveau d'eau côté amont (côté mer) de l'ouvrage, à l'intérieur du massif ou à quelque distance, et à chaque moment du cycle marée, permet d'étudier l'effet de la surpression hydrostatique dans le sol visà-vis d'un éventuel glissement. Cette surpression est due à la forte dénivellation du niveau d'eau à la basse mer. Elle introduit des poussées hydrostatiques déstabilisatrices (Chapon, 1966).

La zone de perturbation par les fluctuations de la nappe causées par la marée est le lieu de changements importants et alternatifs de pression interstitielle. Ce domaine apparaît nettement comme une zone de dépression qui se neutralise à la pleine mer pour devenir une zone de surpression à mi-jusant. C'est ainsi qu'elle perturbe les caractéristiques physiques et même mécaniques du sol.

Le sol subit dans ce mécanisme des vitesses d'écoulement alternatives dues au remplissage et à la vidange cyclique du massif, à un effet dynamique non négligeable. Globalement, les variations de ces vitesses donnent naissance à une accélération d'entraînement qui peut emporter toute la masse de l'ouvrage vers une instabilité par glissement. Localement, ces vitesses sont généralement importantes au pied de ces ouvrages au moment de la basse mer. Elles risquent d'entraîner le matériau lors de leurs sorties, créant ainsi un affouillement. Ce dernier cas est souvent constaté sur les pentes des talus semi-émergés lorsque la mer se retire, lors de la marée basse.

Les résultats obtenus permettent d'approfondir la connaissance de ces phénomènes et d'envisager d'intéressants développements, notamment concernant la stabilité des talus estuariens. Par ailleurs, le modèle physique original que nous avons réalisé peut constituer un outil de base pour d'autres validations. Cette recherche permet ainsi de mieux cerner le domaine de l'aménagement portuaire, qui présente un grand intérêt à la fois scientifique et économique.

\section{Bibliographie}

Alexis A. - Etude géotechnique et sédimentologique de souilles et chenaux de la rade de Lorient. Contribution à la stabilité des sols immergés. Thèse de Docteur Ingénieur, ENSM Nantes, 1987 $260 \mathrm{p}$.

Alexis A., Dos Santos P. Gouraud N. Mazery S, - Réalisation d'un modèle de mesure des écoulements dans un mas. sif en situation estuarienne. Contrat ANVAR Nantes, juin 1990, 79p.

Chapon J. - Travaux maritimes, Tome 2 1966, Ed. Eyrolles pp. 88-91.
De Cazenove E. - Ondes phréatiques sinusoïdales, La houille blanche, $n^{\circ} 7,1971$. pp. 601-315.

Mahé A. - Mécanique des sols. Tome 1 1989. École Centrale de Nantes, pp. 69 99.

Polubarinova P.Y.A. - Theory of ground water movement, 1962, Princeton Ed. $613 \mathrm{p}$.

Rezzoug A., Alexis A. Thomas P. - Ecoulement dans les talus intertidaux. Journal de Recherche Océanographique (accepté à paraitre), 1993'.
Rezzoug A., Alexis A., Thomas P. - Theory and experimental validation of tidal seepage in banks. International Conference on Hydro-Science and Engineering. Washington June 7-11, 19932. Volume 1 part B, pp. 1711-1716.

Rezzoug A. Alexis A. - Impact d'un écoulement cyclique sur un ouvrage semiémergé. Journées nationales de Gènie Civil-Génie Côtier, 26-28 février 1992. Nantes, pp. 486-498. 